\title{
Rigorous analysis of trapped modes in accelerating cavities
}

\author{
Rolf Schuhmann* and Thomas Weiland \\ Darmstadt University of Technology, TEMF, Schlossgartenstrasse 8, 64289 Darmstadt, Germany
}

(Received 11 October 2000; published 20 December 2000)

\begin{abstract}
We report the development of different algorithms for the calculation of quality factors of eigenmodes in accelerating cavities, which have resonance frequencies above the cutoff frequency of the beam tubes. The analysis is based on a discretization of such cavity structures by the finite integration technique, and the radiation at the open boundaries is systematically taken into account by different approaches in time and frequency domain. Results indicate that even single cell cavities of the TESLA type show $Q$ values of $10^{3}$ and multicell cavities values in excess of $10^{4}$. Thus these modes may cause considerable beam instabilities. Comparison with the conventional method of analyzing closed cavities and identifying modes with little change in frequency as a function of boundary condition shows qualitative differences. Some modes from the closed cavity model do not exist in the open structure and thus would be misinterpreted as trapped modes when only a closed cavity analysis is employed.
\end{abstract}

PACS numbers: 02.70.Bf, 02.70.Hm, 41.20.Jb

\section{INTRODUCTION}

Trapped modes in accelerating cavities have been subject to serious consideration with respect to beam instabilities for many years. While the phenomenon of trapped modes is well known in antenna theory, the analysis of these fields remains a complicated task. In the field of accelerator design the typical analysis tools are eigenmode solvers for closed structures such as the $E$ module in MAFIA [1]. Such tools a priori do not allow the rigorous analysis of trapped modes as the basic feature of continuous loss of energy through traveling modes is not included. The eigenmode analysis can give only some hints on the existence of trapped modes. The same facts limit the usefulness of measurements in which typically the beam tubes are electrically short ended and thus also do not take the key issue of traveling modes into account. In many practical cases this analysis is sufficient as it results in upper limits for the impedance of such modes. However, in the case of superconducting structures these upper limits become intolerable and a more detailed analysis becomes indispensable.

In this paper we present several different methods for the analysis of trapped modes in open structures, which are all based on the discretization with the finite integration technique (FIT) [1,2]. The effect of traveling modes is taken into account by different techniques in time or frequency domain.

After the introduction into the basic phenomenon we analyze a single cell cavity with three different methods and show the agreement between them. Finally, we extend the structure to three cells. Even for this still simplified cavity with ideally conducting cavity material we find dipole modes well above the beam tube cutoff frequency with $Q$ values in excess of $10^{4}$.

\footnotetext{
*Email address: schuhmann@temf.tu-darmstadt.de
}

\section{TRAPPED MODES}

Figure 1 shows a model resonator, which, in this case, is similar to the end cell of a TESLA nine-cell structure [3]. The end tubes are assumed to be infinitely long at both sides. We restrict our analysis to modes with an odd azimuthal order $(m=1,3, \ldots$, corresponding to dipole mode, sextupole mode, ...), and thus it is sufficient to discretize one-fourth of the cross section, applying one perfectly electric conducting (PEC) and one perfectly magnetic conducting (PMC) boundary condition at the symmetry planes. Since it is not the aim of this paper to analyze the convergence properties of the fields and the secondary quantities referring to the grid resolution, we use a quite coarse, but fixed discretization with $18 \times 18 \times 37$ nodes $(18 \times 18$ for the cross section, and less than $7 \times 7$ for the beam tube). All results and comparisons refer to this computational model. The field simulations are performed using the program CST MICROWAVE STUDIO [4].

The cutoff frequency of the dipole modes in the beam tubes are shown in Table I. We restrict our analysis in the following to a frequency range of 2.5 to $5.0 \mathrm{GHz}$, because in this range there is only one dipole beam tube mode

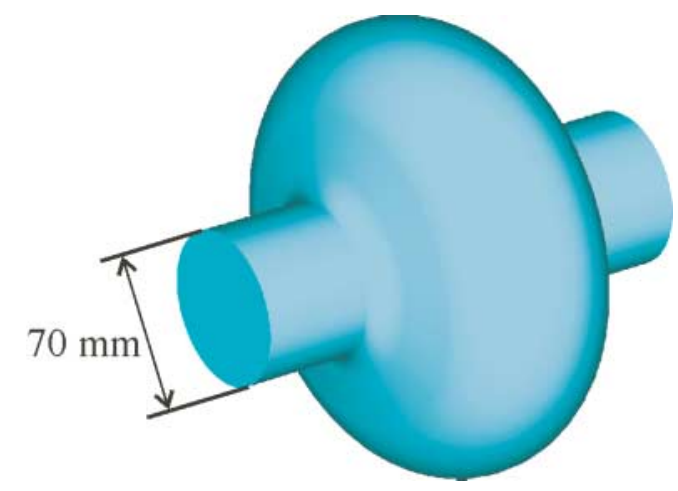

FIG. 1. (Color) A single cell resonator similar to the TESLA cavity with infinitely long beam tubes at both sides. 
TABLE I. Computed [4] cutoff frequency of the dipole (and sextupole) modes in the end tubes (using only $\frac{1}{4}$ of the structure making use of the symmetries). In the frequency range between 2.5 and $5 \mathrm{GHz}$ the deviation between the analytical values and the computational model (fixed discretization with $7 \times 7$ grid points in the cross section of the beam tube) is below $1.2 \%$.

\begin{tabular}{cccc}
\hline \hline Mode & $\begin{array}{c}f / \mathrm{GHz} \\
\text { (simulated) }\end{array}$ & $\begin{array}{c}f / \mathrm{GHz} \\
\text { (analytical) }\end{array}$ & Deviation \\
\hline $\mathrm{TE}_{11}$ & 2.49903 & 2.5097 & $0.43 \%$ \\
$\mathrm{TM}_{11}$ & 5.16461 & 5.2234 & $1.13 \%$ \\
$\mathrm{TE}_{31}$ & 5.61294 & 5.7274 & $2.00 \%$ \\
$\mathrm{TE}_{12}$ & 7.06743 & 7.2680 & $2.76 \%$ \\
\hline \hline
\end{tabular}

above cutoff frequency, and this simplifies the analysis while keeping all the basic physics present ${ }^{1}$. We also do not include sextupole and higher azimuthal modes, as the first cutoff frequency of these modes appears only at $5.61 \mathrm{GHz}$ $\left(\mathrm{TE}_{31}\right)$. The deviation of the simulated cutoff frequency of the $\mathrm{TE}_{11}$ mode to the analytical value is less than $1 \%$ (in spite of the coarse mesh resolution).

If there is a broadband excitation of fields present in the cavity, such as the excitation through a bunched beam current, field energy is deposited over a wide range of frequencies. The part of the spectrum below the cutoff frequency of the first traveling mode in the beam tube cannot leave the cavity. This energy will be stored in true eigenmodes and will only be subject to wall current losses. Thus the $Q$ values of these modes are determined solely by the material of the cavity walls and the field distribution. Typically such $Q$ values - referred to in the following as $Q_{\text {MAT }}$ - are of the order of some $10^{4}$ in copper cavities and some $10^{9}$ in superconducting cavities.

The part of the spectrum above the cutoff frequency of the first traveling mode will lose energy into the walls and also through the ports. However, this radiation loss takes place only at a certain rate per period. Thus there will be associated $Q$ values as well, which we call $Q_{\mathrm{RAD}}$ in the remainder of this paper. Cavity modes with high $Q_{\mathrm{RAD}}$ factors (and thus only weak coupling of the fields to the ports) are identified as "trapped modes."

The total $Q$ value can finally be determined by

$$
\frac{1}{Q_{\mathrm{TOT}}}=\frac{1}{Q_{\mathrm{RAD}}}+\frac{1}{Q_{\mathrm{MAT}}} .
$$

For many practical cases the effect of the contribution due to radiation can be neglected, as the closed cavity analysis yields an upper limit of the final $Q$ value. Whether such a pure eigenmode analysis of a closed cavity model is sufficient with respect to beam dynamics strongly depends on the cavity surface material and the overall accelerator layout.

\footnotetext{
${ }^{1}$ Note that the $\mathrm{TM}_{01}$ and the $\mathrm{TE}_{21}$ modes, which both have cutoff frequencies below $5 \mathrm{GHz}$, cannot exist in this model due to the chosen symmetry conditions at the boundaries.
}

\section{A. Copper structures}

In copper cavities the eigenmode analysis can be used to determine upper values for the $Q$ values of trapped modes. Extensive analysis of such modes has been performed for the $S$ band traveling wave cavities after they were discovered and found to present a serious problem with respect to beam instabilities [5]. Because of the importance of theses modes, special test structures were built that were small enough to be easily computed and large enough to show highly trapped solutions [6]. As the true $Q$ value can only become smaller when radiation losses are added, this analysis was sufficient to be able to design the necessary mode couplers for reducing the $Q$ values.

\section{B. Superconducting structures}

In superconducting cavities the situation is drastically different. Because of extremely small wall losses, $Q$ values determined solely by eigenmode computations yield results that are not tolerable from the beam dynamics point of view. Although it still holds that a closed cavity analysis gives only upper limits, these limits are too high. In this case a careful analysis of trapped modes becomes a crucial issue, as does the design of couplers as the only means to reduce the $Q$ values.

To avoid confusion with the experimental evidence of trapped modes in the TESLA Test Facility (TTF) [7], it should be pointed out here that other phenomena also exist that lead to trapped modes with high $Q$ values. In the TTF case the trapped modes that were found experimentally are most likely to be caused by the effect of the neighbor cavities that did not allow the fields above cutoff frequency to travel away from the main cavity [8]. These trapped modes are externally trapped and thus concise a different subject than the one treated here, where the beam tubes are assumed to be infinitely long with a constant cross section.

\section{CONVENTIONAL EIGENMODE ANALYSIS}

Throughout the rest of this paper, the material losses and $Q_{\text {MAT }}$ are neglected. [However, they can easily be taken into account in the numerical analysis by performing a "power loss" calculation and applying (1).]

We begin with the conventional method of computing true eigenmodes of the closed model cavity, where the beam tubes are terminated with either ideal electric or ideal magnetic walls. The influence of the choice of boundary condition on the mode frequency gives a strong hint for possibly trapped modes, i.e., when the influence is small, the relative field strength at the beam tube ends is small as well (as follows from Slater's theorem).

The results for all calculated dipole modes with resonances between 2.5 and $5 \mathrm{GHz}$ are listed in Table II, also including a coupling factor $K$, that gives a measure for the strength of the influence of the boundary condition. This 
TABLE II. Eigenmodes in the model cavity and the influence of the chosen boundary condition at the beam tube. Only dipole modes are listed. Column "BC" denotes the type of the boundary condition at the beam tube ends $(M$ : perfectly magnetic conducting; $E$ : perfectly electric conducting boundary condition). Modes that depend only weakly on the change in boundary condition are found. Other modes, which differ significantly in mode pattern and frequency between the two cases of beam tube boundary conditions, are not candidates for trapped modes.

\begin{tabular}{|c|c|c|c|c|c|c|c|c|}
\hline $\mathrm{BC}$ & No. & $f_{E} / \mathrm{GHz}$ & $\mathrm{BC}$ & No. & $f_{M} / \mathrm{GHz}$ & $f_{\text {mid }} / \mathrm{GHz}$ & $K=|\Delta f / f|$ & $\log (1 / K)$ \\
\hline E & 3 & 2.611490 & $M$ & 3 & 2.482180 & 2.546835 & 0.051 & 1.3 \\
\hline \multirow[t]{3}{*}{$E$} & 4 & 2.636410 & $M$ & 4 & 2.534320 & 2.585365 & 0.039 & 1.4 \\
\hline & & & $M$ & 6 & 2.847730 & 2.847730 & & \\
\hline & & & $M$ & 7 & 2.999960 & & & \\
\hline E & 6 & 3.018400 & $M$ & 9 & 3.057130 & 3.037765 & 0.013 & 1.9 \\
\hline E & 8 & 3.219310 & $M$ & 11 & 3.591350 & 3.405330 & 0.109 & 1.0 \\
\hline E & 9 & 3.332230 & $M$ & 10 & 3.334890 & 3.333560 & 0.001 & 3.1 \\
\hline E & 10 & 3.472680 & $M$ & 14 & 3.883820 & 3.678250 & 0.112 & 1.0 \\
\hline E & 13 & 4.026830 & $M$ & 17 & 4.150410 & 4.088620 & 0.030 & 1.5 \\
\hline E & 15 & 4.147450 & & & & 4.147450 & & \\
\hline$E$ & 18 & 4.226440 & & & & 4.226440 & & \\
\hline \multirow[t]{2}{*}{$E$} & 19 & 4.279100 & $M$ & 19 & 4.269450 & 4.274275 & 0.002 & 2.6 \\
\hline & & & $M$ & 20 & 4.364460 & 4.364460 & & \\
\hline \multirow[t]{2}{*}{$E$} & 21 & 4.390450 & $M$ & 22 & 4.383840 & 4.387145 & 0.002 & 2.8 \\
\hline & & & $M$ & 24 & 4.660490 & 2.330245 & & \\
\hline$E$ & 22 & 4.587560 & $M$ & 25 & 4.676190 & 4.631875 & 0.019 & 1.7 \\
\hline$E$ & 24 & 4.850230 & & & & 4.850230 & & \\
\hline
\end{tabular}

factor is defined the same as the coupling factor in multicell cavities by

$$
K=2 \frac{\left|f_{\mathrm{M}}-f_{\mathrm{E}}\right|}{f_{\mathrm{M}}+f_{\mathrm{E}}}
$$

where $f_{\mathrm{M}}, f_{\mathrm{E}}$ are the resonance frequencies of the cavity terminated with PMC or PEC walls, respectively. Some of the modes are good candidates for trapped modes, with small and very small coupling factor $K$. Some modes cannot be identified as having similar field patterns in both cases and thus are not likely to represent trapped modes in the open structure.

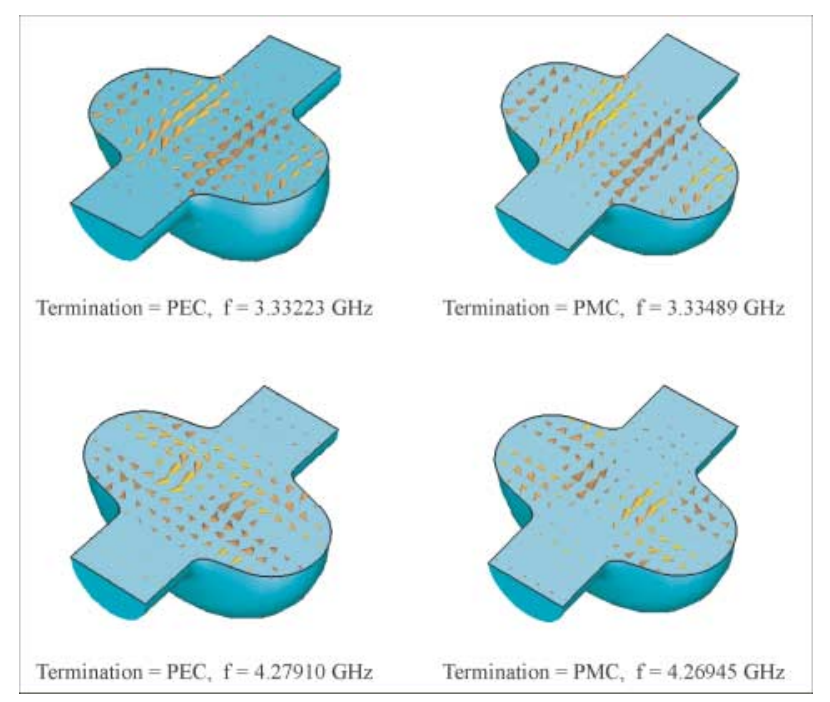

FIG. 2. (Color) Electric fields of two modes near $f=$ $3.33 \mathrm{GHz}$ and $f=4.28 \mathrm{GHz}$, for which the influence of the beam pipe boundary condition (electric or magnetic) is minimal.
The only conclusion from this type of analysis can be that the total $Q$ values for these potentially trapped modes will be lower in the real cavity than the values of $Q_{\text {MAT }}$ computed from the eigenmode field pattern. Figure 2 shows the modes with minimal difference in eigenfrequency as a function of change of boundary condition. These modes obviously have very small field strengths at the boundaries and thus are good candidates for trapped modes.

In superconducting cavities these upper limits calculated from closed cavities are far too high as the $Q$ value is determined almost entirely by the loss due to radiation into the beam tubes. Thus we will investigate open structures in the next section.

\section{TIME DOMAIN ANALYSIS}

A seemingly simple approach to attack the open cavity problem is to use a time domain analysis including waveguide ports. These waveguide boundary ports simulate infinitely long beam tubes and have been successfully used in $S$-parameter computations for waveguide components [9]. As the orthogonality of the waveguide modes in the boundary plane is exactly taken into account (in the sense of the numerical model), this boundary condition is well suited to also deal with high- $Q$ cases with small time signals at the ports.

The $Q_{\mathrm{RAD}}$ values of trapped modes are determined solely by the ratio of the stored energy in the cavity and the power radiating through the boundary ports. Thus, assuming an arbitrary excitation of the cavity and freely oscillating fields, the knowledge of the time signals at the ports, 


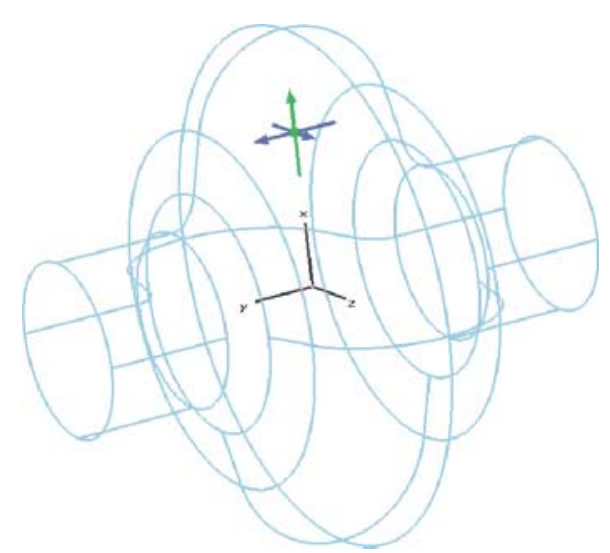

FIG. 3. (Color) Virtual pickup antennas for field components in the inner cavity volume.

which include the exponential decay of the stored energy, is sufficient for the calculation of $Q_{\mathrm{RAD}}$.

In principle, the port modes can be used for both the excitation of the cavity and the detection of the rates of energy decay. However, this approach leads to very long settling times especially for the desired trapped modes. It is more efficient to introduce virtual pickup antennas in the inner volume of the cavity in the simulation, as shown in Fig. 3, and to calculate the transfer function between the signals at the waveguide boundary and the signals at these antennas. According to the reciprocity theorem, the $Q$ values can finally be extracted from this function, no matter which of the signals serves as the input and which serves as the output signal. As the cavity modes may have zero field components at some points, more than one antenna has to be used.

The excitation signal is chosen to be a modulated Gaussian pulse, having a Gaussian shaped spectrum ranging from about 2.5 to $5 \mathrm{GHz}$ with middle frequency $3.75 \mathrm{GHz}$. As shown in Fig. 4, all resonances outside the desired frequency range are only weakly excited and can be neglected (as well as the higher beam tube modes, as mentioned above).

A typical output time signal at one of the pickup antennas is shown in Fig. 5 (left). From a discrete Fourier transform (DFT) of the signals [cf. Fig. 5 (right)], we obtain
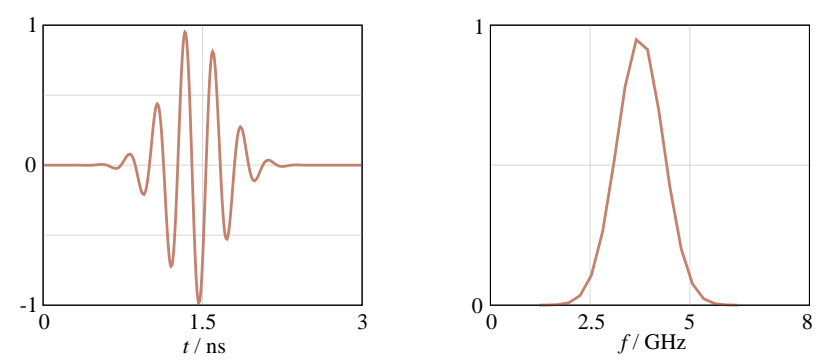

FIG. 4. (Color) Normalized time signal and DFT spectrum of the dipole waveguide mode as used for the excitation of the structure.
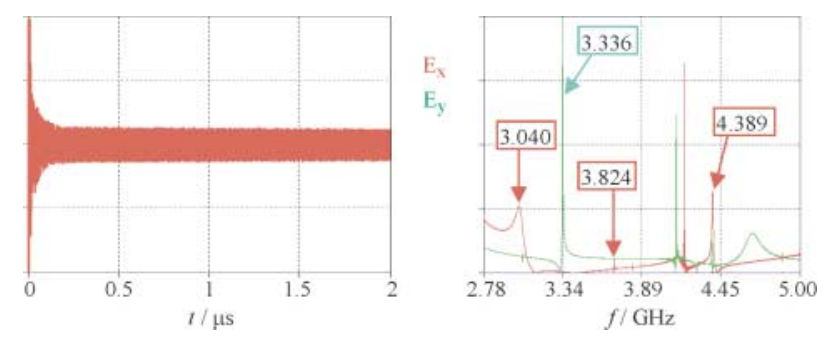

FIG. 5. (Color) Amplitude signal of one of the virtual antennas (left) and spectra of some $E$-field antennas as a result from the DFT (right).

the cavity impedance (the transfer functions between the beam tube ports and the pickup antennas) and thus the width of the trapped resonances. For the cavity analyzed here, the spectrum clearly shows some sharp resonances. From the zoomed plot around $f=3.336 \mathrm{GHz}$ in Fig. 6, we can extract the $3 \mathrm{db}$ bandwidth $\Delta f=0.0022 \mathrm{GHz}$ and thus a quality factor for the corresponding mode of $Q_{\mathrm{RAD}}=f / \Delta f \approx 1514$.

Once the quasiresonant frequencies are known, one can also perform a DFT of the fields in the cavity at those frequencies. The field patterns resulting from such "DFT monitors" show the "true" field of trapped modes, including the radiation at the ports, according to the chosen excitation. These field results can be used to find the correspondences between the modes in the open structure and the results of the conventional analysis with closed boundaries. It turns out that not all eigenmodes in Table II have an analog, if the beam tube is opened. This shows quite clearly that a closed cavity analysis may lead to qualitatively different results. Some candidates for trapped modes, as found in this eigenmode analysis, do not show up at all in the open structure analysis.

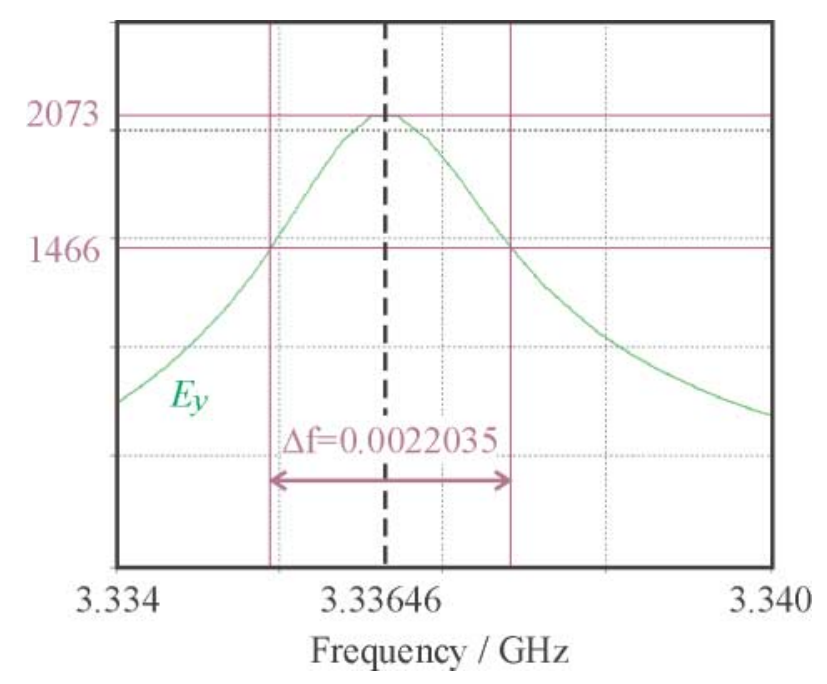

FIG. 6. (Color) Zoomed spectrum of the $E_{y}$ component around its peak at $f=3.33645 \mathrm{GHz}$ showing a $3 \mathrm{db}$ bandwidth of $\Delta f=0.0022 \mathrm{GHz}$ and thus a quality factor of $Q_{\mathrm{RAD}}=1514$. 
One serious problem with the time domain analysis as described so far is that all modes with high $Q_{\mathrm{RAD}}$ factors are related to long settling times, when they are excited by fields at the port modes. Conversely, if they are oscillating in the cavity, the time signals at the ports are very low, and again long simulation times are needed for a numerically robust extraction of the $Q_{\mathrm{RAD}}$ factors. Additionally, if the simulation is aborted before all the energy has left the cavity (as in the example in Fig. 5), the DFT results are overlaid by a si function $[\sim \sin (\omega) / \omega]$, which may lead to wrong results for the $3 \mathrm{db}$ bandwidth and thus for $Q_{\mathrm{RAD}}$.

If we require that the frequency resolution of a signal's spectrum is at least $\Delta f_{\text {sign }}<\frac{1}{2} \Delta f_{3 \mathrm{db}}$ (the bandwidth of the resonance peak), we find, from signal theory, that at least a total number of

$$
n_{\text {min }}=\frac{T_{\text {sim }}}{\Delta t} \geq 2 \frac{Q_{\mathrm{RAD}} / f}{\Delta t}=2 n_{\text {sample }} Q_{\mathrm{RAD}}
$$

time steps are needed in the simulation to resolve an expected $Q$ factor, where $n_{\text {sample }}$ is the number of time steps per period (at least 20 in typical cases). As we can see from this formula, the numerical cost of the time domain analysis increases linearly with $Q$ (which, of course, is a priori unknown).

This situation gets even worse, as in this type of analysis - using a Cartesian mesh model of the cavity, which is not perfectly rotationally symmetric - parasitic resonances with a higher azimuthal order (sextupole and higher modes) are weakly excited. The coupling between these cavity modes and the dipole beam tube mode is due to only numerical (grid) effects and, thus, is very low. This leads to extremely high quality factors and settling times (cf. Fig. 5, with a parasitic peak in the $E_{x}$ spectrum at $f=4.191 \mathrm{GHz}$ ), which overlay the time signals of the desired dipole modes. This problem may be solved by using narrow-banded input signals, but only if the resonance frequencies of the dipole modes and the higher order modes are not too close.

Another problem with the time domain approach is the appropriate location of the antennas, which is required for a good resolution of the resonances in the resulting DFT spectrum. As can be seen from the results presented here (cf. Table III), some resonances may be overseen or appear only with very poor resolutions in the time signals. Moreover, for neighboring peaks with only small differing resonance frequencies, the peaks are often unsymmetrical.

These considerations lead to the assumption that the use of the time domain approach combined with a discrete Fourier transform is not well suited, or at least not very efficient, for the calculation of trapped modes with extremely high values of $Q_{\mathrm{RAD}}$.

The situation gets slightly better if alternative techniques are applied for the spectral analysis of the time signal. In particular, some approaches from filter synthesis or Prony's method [10] are able to make use of parts of the time signals without getting in trouble with the errors intro- duced by the premature abortion of the simulation. However, for the high $Q$ cases to be considered here, these techniques sometimes have numerical stability problems, and often parasitic resonances and $Q$ factors are found.

Therefore, in the next section two approaches in the frequency domain are presented. Based on the same computational model, they again lead to eigenvalue problems, but now include the open boundary condition at the waveguide ports.

\section{FREQUENCY DOMAIN ANALYSIS}

A method to calculate the cavity impedance in the frequency domain, including the radiation at the beam tube ports, has already been presented [11], and, in principle, the $Q$ factors of trapped modes can be derived from these results in a similar way as mentioned above for the time domain scheme (cf. Figs. 5 and 6). However, in this approach an inhomogeneous equation has to be solved for each frequency point, and thus it seems to be more appropriate to pass over to an eigenvalue equation again.

\section{A. Complex eigenvalue formulation}

To this end, the computational model of the FIT discretization including the energy loss through the waveguide boundaries is formulated in frequency domain [12], leading to a linear system of equations for the vector $\hat{\mathbf{e}}$ of electric grid voltages (the state variables of the FIT formulation),

$$
\left(\mathbf{A}_{\mathrm{CC}}^{\prime}+\mathbf{B}_{\mathrm{WG}}(\omega)-\omega^{2} \mathbf{I}\right) \overline{\mathbf{e}}=\mathbf{r}_{\omega} .
$$

The system matrix consists of the operator $\mathbf{A}_{\mathrm{CC}}^{\prime}$ for the (slightly modified) curl-curl-wave equation and a special waveguide boundary operator $\mathbf{B}_{\mathrm{WG}}$, which is complex and nonsymmetrical. On the right-hand side, $\mathbf{r}_{\omega}$ includes the (monochromatic) excitations at the ports.

If there are no excitations present (freely oscillating fields), we obtain the complex and nonlinear eigenvalue problem

$$
\left[\mathbf{A}_{\mathrm{CC}}^{\prime}+\mathbf{B}_{\mathrm{WG}}(\underline{\omega})\right] \widehat{\mathbf{e}}=\underline{\omega}^{2} \widehat{\mathbf{e}} .
$$

Its solutions are the complex eigenvectors $\hat{\mathbf{e}}$ and the corresponding eigenvalues (the squared complex frequencies $\underline{\omega}=\omega+i \alpha)$. The $Q$ factor of these modes finally is given by

$$
Q_{\mathrm{RAD}}=\frac{\omega}{2 \alpha}=\frac{\operatorname{Re}(\omega)}{2 \operatorname{Im}(\omega)} .
$$

To linearize the eigenvalue formulation, the frequency dependence of the matrix $\mathbf{B}_{\mathrm{WG}}$ is neglected and a constant matrix $\mathbf{B}_{\mathrm{WG}}\left(\omega_{0}\right)$ is used instead, where $\omega_{0}$ is a real-valued approximation of the complex eigenfrequency. Especially for the modes with high $Q$ factors, this is a valid simplification, if $\omega_{0}$ is chosen to be the eigenfrequency of the real problem with closed (PMC or PEC) boundaries. Finally, the task is to solve a (still complex and nonsymmetrical) linear eigenvalue problem, where a good start solution for 
TABLE III. Some results of the computation of resonance frequencies (in GHz) and $Q$ factors in the open single-cell test cavity. Time domain: The resonances are extracted from different time signals via graphical evaluation of the DFT or by signal processing techniques (SP). In some cases, no numerically stable results can be found due to too weak signals. Frequency domain: solution of a complex eigenvalue problem and modal approach.

\begin{tabular}{|c|c|c|c|c|c|c|c|c|}
\hline \multirow{2}{*}{\multicolumn{2}{|c|}{$\begin{array}{c}\text { Closed cavity ("M") } \\
\text { (real } E V \text { problem) }\end{array}$}} & \multirow{2}{*}{\multicolumn{3}{|c|}{ Time domain }} & \multicolumn{4}{|c|}{ Frequency domain } \\
\hline & & & & & & & & \\
\hline No. & $f_{\text {res }}$ & Type & $f_{\text {peak }}$ & $Q$ & $\operatorname{Re}\left(f_{\text {res }}\right)$ & $Q$ & $f_{\text {res }}$ & $Q$ \\
\hline 9 & 3.057 & $\operatorname{DFT}\left(E_{x}\right)$ & 3.031 & 71 & & & & \\
\hline & & $\mathrm{SP}\left(E_{x}\right)$ & 3.039 & 47 & 3.036 & 62 & 3.038 & 61 \\
\hline 10 & 3.335 & $\operatorname{DFT}\left(H_{z}\right)$ & 3.336 & 1510 & & & & \\
\hline & & $\mathrm{SP}\left(H_{z}\right)$ & 3.336 & 1538 & 3.334 & 1558 & 3.334 & 1578 \\
\hline 17 & 4.150 & $\operatorname{DFT}\left(E_{y}\right)$ & 4.153 & $\ldots$ & & & & \\
\hline & & SP (all) & $\ldots$ & $\ldots$ & 4.155 & 70 & 4.153 & 69 \\
\hline 19 & 4.269 & $\operatorname{DFT}\left(H_{z}\right)$ & 4.280 & 417 & & & & \\
\hline & & $\operatorname{DFT}\left(H_{x}\right)$ & 4.281 & 441 & & & & \\
\hline & & $\operatorname{SP}\left(H_{x}\right)$ & 4.280 & 444 & 4.276 & 447 & 4.277 & 420 \\
\hline 22 & 4.384 & $\operatorname{DFT}\left(E_{x}\right)$ & 4.391 & 635 & & & & \\
\hline & & $\mathrm{SP}\left(E_{x}\right)$ & 4.391 & 654 & 4.387 & 658 & 4.387 & $>603$ \\
\hline
\end{tabular}

iterative solvers is given again by the solution of the corresponding real problem.

Some results of this procedure for the single cell cavity are listed in Table III, compared to the results from the time domain calculation (where the resonances are evaluated either by a discrete Fourier transformation or by signal processing techniques). The typical relative deviation is below $10^{-3}$ for the resonance frequencies and $10^{-2}$ for the $Q$ factors (except for the DFT results, which suffer from the graphical evaluation and the influence of the finite simulation time). For some of the resonances, accurate results can be obtained only in the frequency domain analysis, due to the weak time signals or their poor resolution in the frequency domain - at least for the pickup antennas used here.

A typical trapped-modes analysis requires one to calculate both several (10-30) solutions of the real problem (with closed boundaries) and some solutions of the complex formulation, and the most severe disadvantage of this approach is the high numerical cost of the complex solvers. Thus, in the next section we present an alternative frequency domain approach, which does not require the solution of a complex eigenvalue problem.

\section{B. Modal approach}

The following "modal" approach is based on a result of Slater's work [13], which has previously been used for a similar analysis [14]. The waveguide-coupled cavity is interpreted as a multiport system, and the impedance matrix $\left(Z_{i j}\right)$ referring to the generalized voltage and current quantities at the ports of this system is calculated. To this end we use a set of modal coefficients, which are related to the $3 \mathrm{D}$ cavity modes and their coupling to the $2 \mathrm{D}$ waveguide modes at the ports. The desired eigenmodes and their external $Q$ factors can then be derived by means of a simple network consisting of the impedances of the structure and the matched resistive loads $Z_{e}$ of infinitely long (reflection-free) waveguides.

The model problem introduced above can be viewed as a one-port system, making use of the third symmetry of the structure, and thus the following derivation concentrates on such simple systems (but can easily be extended to the general case). The impedance matrix then reduces to one single impedance quantity related to the input port of the cavity, which is defined as a reference plane in the beam tube. We introduce a PMC boundary condition at this reference plane and perform a conventional (real-valued) eigenmode analysis of the closed structure as presented above. From Slater [13] we get the following formula for the impedance of the cavity:

$$
\begin{aligned}
Z_{11}(\omega) & =\sum_{a} Z_{a}(\omega) \\
\text { with } Z_{a}(\omega) & =\frac{\nu_{a 1}^{2} /\left(\varepsilon \omega_{a}\right)}{j\left(\omega / \omega_{a}-\omega_{a} / \omega\right)},
\end{aligned}
$$

where $\omega_{a}$ is the resonance frequency of the $a$ th eigenmode of the closed structure and $\nu_{a 1}$ is the expansion coefficient of this mode referring to the two-dimensional waveguide mode in the beam tube (the fundamental mode in the guide with index 1). In the continuous regime of this formula, the summation has to be performed over the infinite number of cavity eigenmodes $(a=1 \cdots \infty)$.

If the cavity (the one-port system) is terminated by an external impedance $Z_{e}$ [where, in our case, this is the characteristic impedance of the beam tube mode $Z_{e}=$ $Z_{01}(\omega)$, we obtain the scalar, nonlinear eigenvalue problem for free oscillations in this small network,

$$
Z_{e}(\underline{\omega})+Z_{11}(\underline{\omega})=0
$$


From the complex eigenfrequencies $\underline{\omega}$ of this equation we find the $Q$ factors of the corresponding modes according to $(6)$.

To use this approach in the discrete model, we have to ensure that the orthogonality properties of both the 3Dcavity modes and the 2D-waveguide modes are fulfilled this is exactly true for the FIT discretization - and that they are properly normalized. The impedance now appears as a finite sum over a number $N$ of cavity modes (corresponding to the finite number of degrees of freedom in the model). However, in practical application, only a limited number, $p=10-500$, of eigenmodes can be calculated, and usually we have $p \ll N$. From the denominator in (7) we can expect to obtain a good approximation of the impedance for such a truncated summation, if $\omega<\omega_{p}$. If we are looking for the complex eigenmode related to the $a$ th real solution, this is usually fulfilled for $p \gg a$.

For a rigorous solution of the nonlinear equation (8), iterative schemes (e.g., Newton) can be applied. In many cases good results can also be obtained using the following simplifications (as proposed by Slater [13]): Typically in the neighborhood of the resonance frequency only one of the addends (with index $a$ ) is strongly varying, and all the others can be assumed to be approximately constant (but not zero),

$$
\begin{aligned}
Z_{11}(\omega) & \approx Z_{a}(\omega)+Z_{a 1} \\
\text { with } Z_{a 1} & =\sum_{i \neq a} Z_{i}\left(\omega_{a}\right)=\text { const. }
\end{aligned}
$$

In a similar manner, the impedance of the waveguide may also be approximated by a constant value $Z_{01}(\omega) \approx$ $Z_{01}\left(\omega_{a}\right)$. A simplified analysis of (8) then yields

$$
Q=\frac{Q_{\mathrm{ext}}}{g}
$$

with the abbreviations

$$
g+j b=\frac{Z_{01}}{Z_{e}+Z_{a 1}} \quad \text { and } \quad Q_{\mathrm{ext}, a 1}=\frac{\varepsilon \omega_{a} Z_{01}}{\nu_{a 1}^{2}} .
$$

The key point in the modal approach is the control of the truncation error due to the finite number of cavity modes, which can be calculated with reasonable computer resources. Once the $p$ real eigenmodes of the closed structure have been calculated, the series $Q(i)$ of $Q$ factors (using $i=1, \ldots, p$ modes) can be easily evaluated. For the convergence properties of this series we find that (a) the value of $Q_{\mathrm{ext}, a 1}$ from (11), where no additional modes are needed, is always a lower bound for the final $Q$ factor and (b) if $\operatorname{Im}\left(Z_{a 1}\right)>0$ for a number $p_{0}$ of modes (this can be easily checked), we get an improved lower bound by $Q\left(p_{0}\right)=Q_{\mathrm{ext}} / g\left(p_{0}\right)$, as $Q(p)$ is monotonously increasing for $p>p_{0}$.

In many cases, these two results and the convergence curve $Q(p)$ (the computed $Q$ factor vs the number of considered modes) allow an estimation of the final value of the $Q$ factor. The number of modes needed for an accurate solution depends (among others) on the density of resonances in the real spectrum.

As an example, Fig. 7 shows the convergence of $Q(p)$ for the 10th mode in the single cell cavity: The "final" values of the modal approach (real part of the resonance frequency $f_{10}=3.334 \mathrm{GHz}$ and $Q \approx 1578$ ) is in very good agreement with the results of the complex eigenvalue formulation presented in the previous section (cf. Table III). A first approximation of this value can already be obtained by the evaluation of $Q_{\text {ext,10 }} \approx 1226$ (where no further modes are needed), and for a relative deviation below 10\% about 90 modes have to be taken into account. The results from an exact solution of (8) and the simplified formula in (10) are in nearly perfect agreement (indistinguishable curves in Fig. 8).

Note that obviously many modes do not contribute considerably to the result for $Q$ (e.g., the modes between $p=90$ and $p=97)$. These are modes with a higher azimuthal order ${ }^{2}$ and thus no (or only an extremely weak) coupling to the dipole beam tube mode at the input port. As a consequence, the number of modes needed in the modal approach can be considerably decreased, if only dipole modes are calculated (by use of a 2D mesh for rotationally symmetric structures and a modification of the system matrix [1]).

The results for some other modes are summarized in Table III. For the resonant mode, No. 22, at $f_{\text {res }}=4.387 \mathrm{GHz}$ the modal sum has not yet converged very well; however, a lower limit $Q>603$ is available.

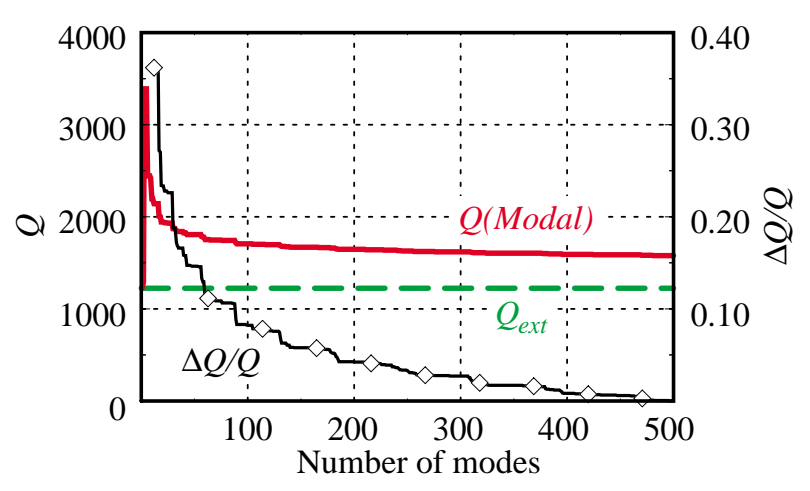

FIG. 7. (Color) Convergence curve of the calculation of the $Q$ value of mode No. $10(f=3.3344 \mathrm{GHz})$ using the modal approach. Related to the "final" value $Q=1578$ (including 500 cavity modes), we need about 90 modes to obtain a relative accuracy of $10 \%$. The (easy accessible) value $Q_{\text {ext }}$ from (11) is a lower bound for $Q$.

\footnotetext{
${ }^{2}$ These cavity modes, which only couple due to the nonsymmetric computational grid, deteriorate the time domain analysis as mentioned above. In the frequency domain they do not significantly contribute to the $Q$ calculation.
} 


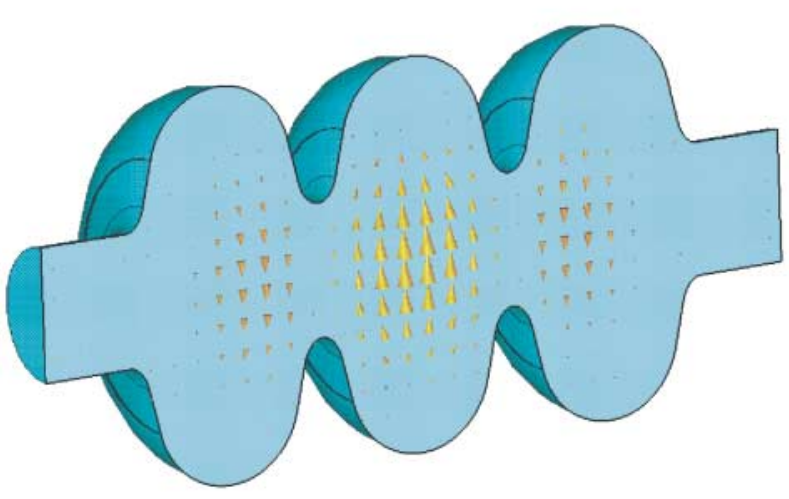

FIG. 8. (Color) Eigenmode in a three-cell cavity with resonance frequency $f=3.0700 \mathrm{GHz}$ (electric field).

Note that, due to the dispersion effects of the time stepping scheme, the resonance frequencies in the time domain approach are always slightly higher than the results from frequency domain.

\section{THREE-CELL CAVITY}

To investigate the influence of the number of cells to the quality factor $Q_{\mathrm{RAD}}$ of the dipole modes, we consider the cavity in Fig. 8, which consists of three cells and a beam tube of the same type as in the simple model above. From the eigenmode computation with closed PMC boundaries, some dipole modes can be found in the frequency range between 2.5 and $5 \mathrm{GHz}$, and thus above the beam tube cutoff. One of these modes with resonance frequency $f=$ 3.0700 GHz is also shown in Fig. 8 .

For the open structure we at first perform a time domain analysis with as much as $2 \times 10^{6}$ time steps and a sampling rate in time of $n_{\text {sample }}=1 / f \Delta t \approx 50$ according to Eq. (3). For the signal spectrum this leads to a resolution of $\Delta f_{\text {sign }} \approx 80 \mathrm{kHz}$, and in the result in Fig. 9 the resonance peak is only quite poorly resolved. As a consequence, the $Q$ factor obtained by this approach cannot be expected to have a very good accuracy. A graphical evaluation only yields the estimation $Q_{\mathrm{RAD}}>20000$.

From the frequency domain approaches we get the results as summarized in Table IV. The resonance frequencies are in very good agreement, and the $Q$ values differ in the range of about $2 \%$. This deviation can be explained with the still not fully converged results: In the modal approach the series $Q(p)$ is still slightly decreasing

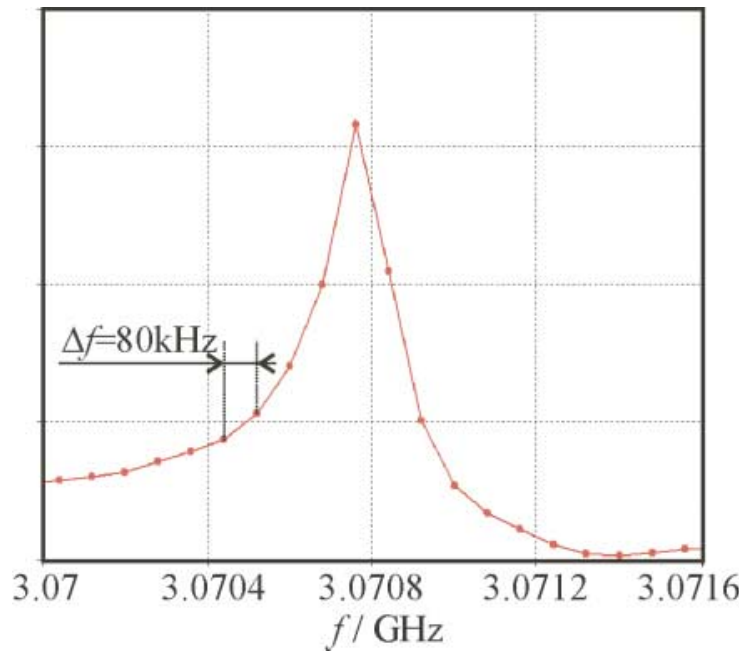

FIG. 9. (Color) DFT spectrum of the time domain analysis of the three-cell cavity. After about $2 \times 10^{6}$ time steps the spectral resolution is about $80 \mathrm{kHz}$.

after $p_{\max }=500$ modes, and in the complex eigenmode calculation an extremely good accuracy of the eigenvalue is required, as its real and imaginary parts differ by several orders of magnitude.

A similar result can be obtained for another trapped mode at $f=3.3421 \mathrm{GHz}$ (cf. Table IV).

\section{COMPARISON}

As shown for the small test example and the three-cell cavity, all three approaches for the calculation of external quality factors yield comparable results, showing relative deviations of some percent for the $Q$ factors.

The time domain approach can be considered to be principally the most rigorous and exact method, as no further simplifications are made within the computational model. However, it suffers from long simulation times and the difficulties with the graphical evaluation of the DFT results, or with the numerical stability of the signal processing techniques, respectively.

Also, in the modal approach, no further approximations are made, except for the truncation of the sum in the impedance formula. Thus, reliable results can be expected from this algorithm, whenever the series of $Q$ factors seems to have converged for a specific complex eigensolution. The computational cost of the real eigenvalue analysis (requiring up to several hundreds of field solutions) might also be quite high; however, as a benefit we

TABLE IV. Some resonance frequencies (in GHz) and $Q$ factors in the open three-cell cavity.

\begin{tabular}{lccccccc}
\hline \hline \multicolumn{2}{c}{$\begin{array}{c}\text { Closed cavity ("M") } \\
\text { (real } E V \text { problem) }\end{array}$} & \multicolumn{2}{c}{ Time domain } & \multicolumn{3}{c}{ Complexuency domain } \\
No. & $f_{\text {res }}$ & $f_{\text {peak }}$ & $Q$ & $\operatorname{Re}\left(f_{\text {res }}\right)$ & $Q$ & $f_{\text {res }}$ Modal & $Q$ \\
\hline 22 & 3.0700 & 3.0708 & $>20000$ & 3.0697 & 25579 & 3.0697 & 26100 \\
26 & 3.3422 & 3.3435 & $>12500$ & 3.3421 & 13489 & 3.3421 & 13934 \\
\hline \hline
\end{tabular}


obtain field solutions of all real eigensolutions in the cavity, which often is of special interest at least for the lower modes.

The approach using the complex eigenvalue formulation does not have this convergence problem, as only one eigenmode has to be computed for each $Q$ factor. However, the solution of a complex nonsymmetrical system is still a severe task for the algebraic solver to be employed. Especially for the high- $Q$ case of trapped modes, the calculated eigenvalue must have a very good accuracy for both its real and imaginary parts.

\section{CONCLUSIONS}

We calculate the quality factors of trapped dipole modes in accelerating cavities, which have resonance frequencies well above the cutoff frequency of the beam tube. Three different approaches have been presented, where the radiation at the ports is rigorously taken into account. As these approaches follow quite different paths, the calculated $Q$ factors with deviations in the range of some percent can be considered to be reliable results.

Quantitative results show $Q$ values of 1000 for single cell cavities and more than 10000 for three-cell cavities. Thus the impedance above cutoff frequency may yield a serious contribution to beam instabilities.

The conventional procedure of computing closed cavity modes and comparing the eigenfrequencies when changing the beam tube end boundary condition may give hints for the existence of trapped modes. This analysis yields upper limits for the contribution of the fields above cutoff frequency, which may be sufficient in some practical applications. However, a comparison between closed cavity analysis and open cavity analysis shows that not all modes that appear to be candidates for trapped modes from the closed cavity analysis are actually real trapped modes. Thus, for some modes, the closed cavity analysis does give upper limits. However, for some modes, this type of analysis may give wrong results.

Especially for superconducting accelerators, the closed cavity approximation yields unacceptable values for the impedance, and a rigorous analysis of the open cavity structure is indispensable.

[1] T. Weiland, Part. Accel. 15, 245 (1984).

[2] T. Weiland, Int. J. Numer. Model. 9, 295 (1996).

[3] DESY Report No. 1997-048, 1997, edited by R. Brinkmann, G. Materlik, J. Rossbach, and A. Wagner.

[4] CST MICROVAVE STUDIO is available from CST GmbH, Buedinger Strasse 2a, 64289 Darmstadt, Germany (www.cst.de).

[5] S Band Collider Group, T. Weiland et al., Status Report of a $500 \mathrm{GeV} \mathrm{S}$ Band Linear Collider (World Scientific, Singapore, 1992), p. 663.

[6] B. Krietenstein, O. Podebrad, U. v. Rienen, T. Weiland, H.-W. Glock, P. Hülsmann, H. Klein, and M. Kurz, in Proceedings of the Particle Accelerator Conference, Dallas, Texas, 1995 (IEEE, Piscataway, NJ, 1996), p. 695.

[7] S. Fartoukh et al., in Proceedings of the 1999 Particle Accelerator Conference, New York (IEEE, Piscataway, NJ, 1999), p. 922.

[8] N. Baboi, M. Dohlus, C. Magne, A. Mosnier, O. Napoly, and H.-W. Glock, in Proceedings of the 7th European Particle Accelerator Conference, Vienna, 2000 http://accelconf.web.cern.ch/AccelConf/e00/index.html, p. 1107.

[9] M. Dohlus, P. Thoma, and T. Weiland, in Proceedings of the 2nd International Workshop on Discrete Time Domain Modelling of Electromagnetic Fields and Networks, Berlin, 1993 (unpublished).

[10] P. Thoma and T. Weiland, in Computational Accelerator Physics, edited by Robert Ryne, AIP Conf. Proc. No. 297 (AIP, New York, 1994), p. 66.

[11] U. v. Rienen and T. Weiland, in Proceedings of the European Particle Accelerator Conference, Rome, 1988 (DESY, Hamburg, 1988), p. 890.

[12] R. Schuhmann, M. Clemens, P. Thoma, and T. Weiland, in Proceedings of the 12th Annual Review of Applied Computational Electromagnetics Conference, Monterey, 1996 (Applied Computational Electromagnetics Society, Monterey, CA, 1999) p. 1295.

[13] J.C. Slater, in Microwave Electronics (Van Nostrand, Princeton, NJ, 1950).

[14] M. Dohlus, R. Schuhmann, and T. Weiland, Int. J. Numer. Model. 12, 41 (1999). 